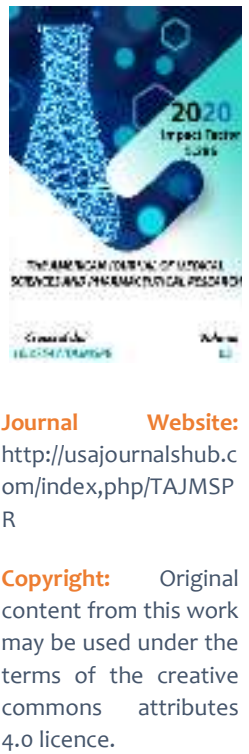

\title{
Clinical And Epidemiological Features Of Bacterial Vaginosis
}

Rakhmatullaeva M.M.

Bukhara State Medical Institute, Bukhara, Uzbekistan

\section{ABSTRACT}

The review article presents the epidemiological characteristics of bacterial vaginosis, data on the lability of the composition of the vaginal microflora, including the species number of lactobacilli, depending on the ethnicity of the woman and the geographical area of residence. Literature data on factors affecting the composition of vaginal microbiocenosis, the most frequently detected types of bacteria in bacterial vaginosis, are presented.

\section{KEYWORDS}

Bacterial vaginosis; vaginal microbiocenosis; lactobacilli; factors affecting the composition of vaginal microbiocenosis.

\section{INTRODUCTION}

Violation of the vaginal ecosystem in women of reproductive age is one of the most urgent problems in obstetric and gynecological practice. The significance of bacterial vaginosis is primarily due to the wide prevalence of this disease. The frequency of detection of BV worldwide varies widely - from $7 \%$ to $68 \%$ [14] and depends on the studied population [13].

In outpatient gynecological practice, BV is found in $15-25 \%$ of patients, among pregnant women, BV occurs in $20-46 \%$ of cases, in women with sexually transmitted infections-up 
to $40 \%$, in women with pelvic inflammatory diseases-in 35\% [9], in pathological whites - up to $87 \%$ [8]. Statistically significant, BV is more common in women under 25 years of age. Moreover, according to the data of Lipova E. V. et al. (2012), in the population with a full examination according to the Amsel criteria, this disease can be detected in every 4 women. In the study of a more modern, moleculargenetic method for diagnosing the composition of vaginal microbiocenosis, BV was detected in $19.7 \%$ of clinically healthy women of reproductive age [2].

It is an indisputable fact that most vaginal infections are of a mixed nature. The share of mixed infections is up to half of cases among all infections of the female reproductive system [8]. However, isolated bacterial vaginosis is rarely diagnosed, since in most cases it is characterized by mixed clinical forms with aerobic microflora and genital candidiasis [8].

The vaginal microflora of most women in different periods of life is quite stable. However, the qualitative and quantitative composition of the vaginal microflora can constantly change in response to exogenous and endogenous factors. The composition of the vaginal microbiocenosis of women of reproductive age is influenced by the phases of the menstrual cycle, hormonal changes in the postmenopausal period, pregnancy, hygiene habits, and sexual activity [9].

Moreover, the specific and quantitative composition of the vaginal microflora depends on the ethnicity of the woman and the geographical area of residence. The predominance of lactobacilli in the vaginal microflora was observed in $89.7 \%$ of European, $80.2 \%$ of Asian, $61.9 \%$ of African and $59.6 \%$ of Latin American women [17]. At the same time, a difference in the species composition of lactobacilli was revealed. In most populations,
Lactobacillus crispatus is the dominant species, and in Caucasian women, Lactobacillus crispatus and/or Lactobacillus jensenii are more common than any other Lactobacillus species [15], whereas vaginal microbiocenosis with the dominance of facultative and obligate anaerobes is more typical for Negroid women (45-55\%), compared to Caucasian (5-15\%) [17]. These same data echo the results of a more recent study, which shows that the overall occurrence of Lactobacillus among African women is significantly lower than in Asian and European women, and the species Lactobacillus vaginalis is the most common species among African women [13].

\section{MATERIALS AND METHODS}

Special attention should be paid to the identification of two age-related peaks in the incidence of BV - in young women aged 18-25 years and in women after 40 years. The first peak may be associated with higher sexual activity at this age, and the second with the onset of hormonal menopausal changes in women of this age [9].

$\mathrm{BV}$ is one of the most pressing health problems around the world, as it causes serious complications related to women's reproductive health. A characteristic feature of $\mathrm{BV}$ is the absence of signs of an inflammatory response of the mucous membrane, which is why many of these women do not have symptoms. The classic course of BV occurs only in a third of patients [2]. The infection usually causes discomfort in the vagina, an unpleasant odor, abnormal discharge and irritation of the vulva. In women with bacterial vaginosis, the disease is not always limited to the lower genital tract. Ascending infection leads to the development of inflammatory diseases of the cervix (cervicitis), as well as the uterus (endometritis) and its appendages (salpingooforit) [11]. In patients with BV, the 
frequency of infectious complications after performing certain gynecological manipulations (abortion, hysteroscopy, laparoscopy) is significantly increased. Unsuccessful attempts at in vitro fertilization are more common in women with concomitant $\mathrm{BV}[12]$.

$\mathrm{BV}$ is also a cause of complications and adverse pregnancy outcomes (miscarriage, chorioamnionitis, birth of small children) and diseases of the postpartum period. It was found that the bacteria associated with BV are more often detected in the amniotic fluid in women with preterm birth and rupture of the fetal membranes and infectious complications of pregnancy [6]. In addition, BV has been shown to increase the risk of infection with HIV, Neisseria gonorrhoeae, Clamydia trachomatis, and Human herpes virus [16]. It should be noted separately that violations of the vaginal microflora are considered a cofactor of the development of cervical neoplasia ( $\mathrm{CIN})$, which is associated with a certain role of the state of vaginal microbiocenosis in susceptibility to human papillomavirus [7].

The state of vaginal microbiocenosis is influenced by factors such as lifestyle and lifestyle, sex education, hygiene habits, occupational hazards, and the use of contraceptives. There is a direct relationship between the incidence of BV and the model of sexual behavior. BV is usually characteristic of women who have a large number of sexual partners, who practice non-traditional sex, and who started their sexual life at a young age [10].

Although only a few risk factors have been identified for BV, the epidemiology of $B V$ is complex and the study of this issue has traditionally focused heavily on the study of sexual behavior. In the literature on the epidemiology of BV and Gardnerella vaginalis, one of the leading microorganisms associated with BV, many aspects have recently been comprehensively considered in the context of sexual behavior. Although BV is not a sexually transmitted infection, sexual transmission is evidenced by the Association of the disease with a number of risk factors associated with sexually transmitted infections. Thus, Turner A. $N$. et al. (2016) showed the existence of an Association of unprotected sex with recurrent BV [19]. Furthermore, BV is much more common in women who have had more than one sexual partner in the past 6 months than in women who have not had a single partner or who have had a single partner [9]. A large number of sexual partners is a risk factor for STIs and supports the sexual route of transmission of BV.

Using the methods of detection of biofilms of A. Swidsinski et al. (2010) studied possible ways to transmit BV [18]. When women with BV were examined, the associated form of Gardnerella vaginalis was detected simultaneously with their partners, indicating that Gardnerella vaginalis was transmitted as a biofilm. The authors concluded that the biofilm (related form) of Gardnerella vaginalis acts as an infectious agent in BV - a disease that has a sexual transmission pathway and involves both women and men and their sexual partners in the infectious process, while dispersed (planktonic) forms of Gardnerella vaginalis do not have a pronounced clinical significance. At the same time, the bound and dispersed forms do not pass into one another and represent independent forms of existence of Gardnerella vaginalis [18].

Microorganisms associated with BV can be isolated from the urethra of men who are sexual partners of patients with BV; sometimes they can cause urethritis, which is manifested 
by burning and pain when urinating. In this case, treatment of the partner is necessary, but this does not prevent the relapse of the disease in women [9].

The analysis of indicators of mucosal microbiocenosis in men carried out by Kostyuk S. A. et al. (2015) revealed a statistically significant increase in the number of the same BV-associated microorganisms as in women, with the exception of Staphylococcus spp. [3]. As you can see, infection occurs: female-male, as well as male-to-female transmission of Gardnerella vaginalis.

\section{RESULT AND DISCUSSION}

Different types of lactobacilli play an important role in maintaining the constancy of the vaginal microflora, but their share in this does not have the same degree. The dominance of Lactobacillus crispatus in the lactoflora is associated with the normal state of the vaginal microbiota, whereas the proportion of Lactobacillus iners-dominated samples in the vaginal lactoflora is higher in dysbiotic processes [1]. The question of the protective capacity of Lactobacillus iners remains controversial. Many sources suggest that the predominance of this type of Lactobacillus in the vaginal microflora increases the risk of developing BV [4].

In bacterial vaginosis, the number of lactobacilli producing hydrogen peroxide significantly decreases or they are completely absent [1], polymicrobial, mainly anaerobic microflora prevails (the proportion of Gardnerella vaginalis, Mycoplasma hominis, Mobiluncus spp. increases), the overall degree of colonization of the vagina by various microorganisms increases (up to 109-1011 CFU/ml), the $\mathrm{pH}$ of the vaginal discharge increases (more than 4.5) [2].
BV is characterized by a large variety of microflora, but it has not yet been proven that any particular microorganism or group of microorganisms plays a role in the development of the disease. Gardnerella vaginalis is the most common bacterial species in BV and has the highest virulence. The main virulence factors of Gardnerella vaginalis are cytotoxicity, the ability to produce the enzyme sialidase, adhesion to epithelial cells, and the ability to form bacterial films.

The second most common type of bacteria after Gardnerella vaginalis was Prevotella. The proportion of gram-negative microorganisms Prevotella spp. among all anaerobes isolated from BV-positive women is about 44-78\% [15]. In recent years, new bacteria associated with BV have been identified, such as Atopobium vaginae, Megasphaera spp., Eggertella spp., Leptotrichia spp., Dialister spp., Slackia spp., BVAB-1, BVAB-2, BVAB-3, Sneathia spp., and others [17].

Atopobium vaginae is rarely isolated in the absence of Gardnerella vaginalis [10], which indicates a synergy between these microorganisms. The ineffectiveness of treatment and frequent relapses of BV are due to the presence of this bacterium in the vaginal microflora.

According to Shchypitsina E. V. et al. (2016) DNA of Gardnerella vaginalis and Atopobium vaginae was detected in 93 and $83 \%$ of women with $\mathrm{BV}$, respectively. Both microorganisms were also found in many women with normal vaginal microflora (in 52 and 38\% of cases, respectively), but the concentration of these bacteria in vaginal samples in women with normal was significantly lower than the concentration in samples in women with BV [9]. 
In BV, Mobiluncus spp./Corynebacterium spp. (95.2\%) and Megasphaera spp./Veillonella spp./Dialister spp. (92.9\%) are also present in significant quantities. Mycoplasma hominis is detected in the vaginal discharge of women with BV less frequently than Ureaplasma spp. [5].

As you can see, BV is associated with a huge range of bacteria. This variety significantly complicates the treatment of $\mathrm{BV}$, reduces its effectiveness and contributes to relapse, the frequency of which reaches up to $30 \%$ within 3 months and $60-80 \%$ of cases after 9 months of treatment $[4,7]$.

\section{CONCLUSION}

Thus, the problem of bacterial vaginosis is extremely relevant due to the wide spread, frequent recurrence of the disease, high probability of complications in obstetric and gynecological practice, which requires further research to expand the understanding of the mechanisms of bacterial vaginosis and improve therapy methods.

\section{REFERENCES}

1. Budilovskaya O. V., Shipitsyna E. V., Gerasimova E. N. et al. Species diversity of vaginal lactobacilli in normal and dysbiotic conditions // Journal of obstetrics and women's diseases. - 2017. - Vol. 66., No. 2. P. 24-32.

2. Voroshilina E. S., Zornikov D. L., Plotko E. E. Normal state of vaginal microbiocenosis: assessment from subjective, expert and laboratory points of view // Vestnik RSMU. - 2017. - No. 2. - P. 42-46.

3. Kostyuk S. A., Shimanskaya I. G., Rudenkova T. V. et al. Study of the specific composition of microflora of urogenital tract mucosa in patients with bacterial vaginosis and their sexual partners // Medical news. - 2015. - No. 5. - P. 46-50.

4. Menukhova Yu. N. Bacterial vaginosis: etiopathogenesis, clinical and laboratory features / / Journal of obstetrics and women's diseases. - 2013. - Vol. LXII., No. 4. - P. 79-87.

5. Nazarova V. V., Shipitsyna E. V., Shalepo K. V., Savicheva A.M. Bacterial communities forming the microecosystem of the vagina in normal and bacterial vaginosis / / Journal of obstetrics and women's diseases. - 2017. - Vol. 66. - No. 6. - Pp. 30-43.

6. Rakhmatullayeva M. M. Treatment of bacterial vaginosis in early pregnancy / / Pharmateka. - 2017. - №12. - P. 67-68.

7. Rakhmatullayeva M. M. Treatment of bacterial vaginosis: problems and prospects / / Pharmateka. - 2019. - Vol. 26. No. 6. - P. 79-83.

8. Sokolova T. M., Marinkin I. O., Kuleshov V. M., Makarov K. Yu. Experience of using a new combined drug for the treatment of urogenital infections / / Obstetrics and gynecology: news opinions, training. - 2019. - Vol. 7., No. 2.-Pp. 68-72.

9. Shipitsina E. V., Khusnutdinova T. A., Ryzhkova O. S. et al. Microbiological, behavioral and clinical-anamnestic predictor factors of bacterial vaginosis in women with secretions / / Journal of obstetrics and women's diseases. - 2016. Vol. 65., No. 3.-P. 32-42.

10. Bradshaw C.S., Walker S.M., Vodstrcil L.A. et al. The influence of behaviors and relationships on the vaginal microbiota of women and their female partners: The WOW health study. J. Infect. Dis. 2014; 209: 1562-1572.

11. Green K.A., Zarek S.M., Catherino W.H. Gynecologic health and disease in relation to the microbiome of the female 
reproductive tract. Fertil Steril. 2015; 104(6): 1351-1357.

12. Haggerty C.L., Totten P.A., Tang G. et al. Identification of novel microbes associated with pelvic inflammatory disease and infertility. Sex Transm Infect. 2016; 92(6): 441-446.

13. Jespers V., Crucitti T., Menten J. et al. Vaginal Biomarkers Study Group. Prevalence and correlates of bacterial vaginosis in different sub-populations of women in sub-Saharan Africa: a crosssectional study. PLoSOne. 2014; 9(10): e109670.

14. Kenyon C., Colebunders R., Crucitti T. The global epidemiology of bacterial vaginosis: a systematic review. Am J. Obstet. Gynecol. 2013; 209(6): 505-523.

15. Lamont R.F., Sobel J.D., Akins R.A. et al. The vaginal microbiome: new information about genital tract flora using molecular based techniques. Br. J. Obstet. Gynaecol. 2011; 118 (5): 533-549.

16. Nardis C., Mosca L., Mastromarino P. Vaginal microbiota and viral sexually transmitted diseases. Ann. Ig. 2013; 25(5): 443-456.

17. Ravel J., Gajer P., Abdo Z. et al. Vaginal microbiome of reproductive-age women. Proc. Natl. Acad. Sci. USA. 2011; 108: 46804687.

18. Swidsinski A., Doerffel Y., Loening-Baucke V. et al. Gardnerella biofilm involves females and males and is transmitted sexually. Gynecol Obstet Invest. 2010; 70(4): 256-263.

19. Turner A.N., Carr Reese P., Snead M.C. et al. Recent biomarker-confirmed unprotected vaginal sex, but not self-reported unprotected sex, is associated with recurrent bacterial vaginosis. Sex Transm Dis. 2016; 43(3): 172-176. 